\title{
Pscroph, a parasitic plant EST database enriched for parasite associated transcripts
}

\author{
Manuel J Torres ${ }^{1,2}$, Alexey A Tomilov ${ }^{1,3}$, Natalya Tomilova1,3, \\ Russell L Reagan ${ }^{1}$ and John I Yoder*1
}

Address: ${ }^{1}$ Department of Plant Sciences, University of California, Davis, Davis, CA 95616, USA, ${ }^{2}$ Plant Genome Mapping Laboratory, Center for Applied Genetic Technologies, 111 Riverbend Road \#224, University of Georgia, Athens, Georgia 30602, USA and 3Vavilov Institute of General Genetics, Russian Academy of Sciences, Gubkina Str., 3, Moscow, Russia

Email: Manuel J Torres - mjtorres@uga.edu; Alexey A Tomilov - tomilov@vegmail.ucdavis.edu;

Natalya Tomilova - tomilov@vegmail.ucdavis.edu; Russell L Reagan - rssllr@yahoo.com; John I Yoder* - jiyoder@ucdavis.edu

* Corresponding author

Published: 16 November 2005

BMC Plant Biology 2005, 5:24 doi:1 0.1 | 86/ | 47|-2229-5-24
Received: 28 July 2005

Accepted: 16 November 2005

This article is available from: http://www.biomedcentral.com/I47I-2229/5/24

(c) 2005 Torres et al; licensee BioMed Central Ltd.

This is an Open Access article distributed under the terms of the Creative Commons Attribution License (http://creativecommons.org/licenses/by/2.0), which permits unrestricted use, distribution, and reproduction in any medium, provided the original work is properly cited.

\begin{abstract}
Background: Parasitic plants in the Orobanchaceae develop invasive root haustoria upon contact with host roots or root factors. The development of haustoria can be visually monitored and is rapid, highly synchronous, and strongly dependent on host factor exposure; therefore it provides a tractable system for studying chemical communications between roots of different plants.
\end{abstract}

Description: Triphysaria is a facultative parasitic plant that initiates haustorium development within minutes after contact with host plant roots, root exudates, or purified haustorium-inducing phenolics. In order to identify genes associated with host root identification and early haustorium development, we sequenced suppression subtractive libraries $(\mathrm{SSH})$ enriched for transcripts regulated in Triphysaria roots within five hours of exposure to Arabidopsis roots or the purified haustorium-inducing factor 2,6 dimethoxybenzoquinone. The sequences of over nine thousand ESTs from three SSH libraries and their subsequent assemblies are available at the Pscroph database http://pscroph.ucdavis.edu. The web site also provides BLAST functions and allows keyword searches of functional annotations.

Conclusion: Libraries prepared from Triphysaria roots treated with host roots or haustorium inducing factors were enriched for transcripts predicted to function in stress responses, electron transport or protein metabolism. In addition to parasitic plant investigations, the Pscroph database provides a useful resource for investigations in rhizosphere interactions, chemical signaling between organisms, and plant development and evolution.

\section{Background}

Parasitic plants directly invade and rob nutrients from host plants $[1,2]$. The consequences can be devastating to the host plant and some of the world's most pernicious agricultural pests are parasitic weeds [3]. The number of parasitic angiosperms is surprisingly large with over four thousand parasitic species identified in nineteen different plant families [4]. Parasitic plants have a wide diversity of growth habits ranging from the tiny flowered mistletoes that live in the tops of trees to the enormously flowered and rootless Rafflesia whose entire vegetative body is endophytic [4]. The degree to which parasites rely on host 
resources also varies. Some obligate parasites, like Rafflesia, have lost photosynthetic capabilities and are fully heterotrophic. Others, like Triphysaria, are facultative parasites that can mature without a host plant but will parasitize neighboring plants when available.

The single feature shared by all parasitic plants is the ability to invade host tissues via a haustorium [1]. Haustoria of parasitic plants fulfill multiple functions including host attachment, penetration, and translocation of resources from host to parasite [5]. Interestingly, the competence to develop haustoria has originated in autotrophic ancestors multiple times during the evolution of angiosperms [6]. There are two general hypotheses for the evolutionary origins of haustoria. One hypothesis suggests that the genes encoding haustorium development are derived from nonplant organisms, such as bacteria or fungi, that are endophytic or which have transferred a set of genes required for haustorium formation into the parasite genome [7]. The second is that genes encoding haustorium development

\section{A}

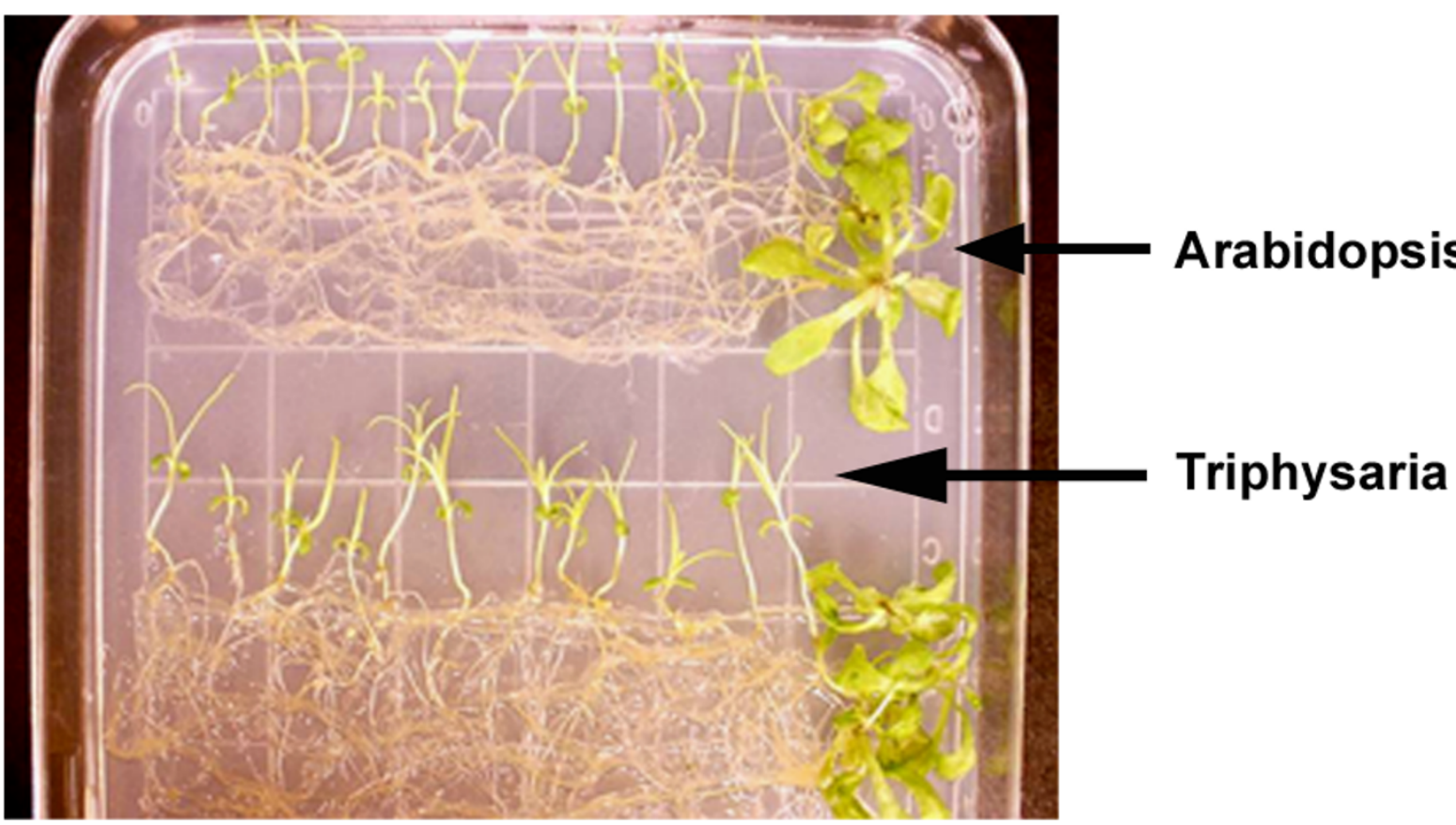

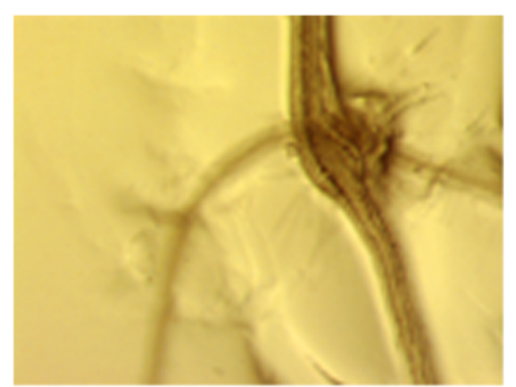

B

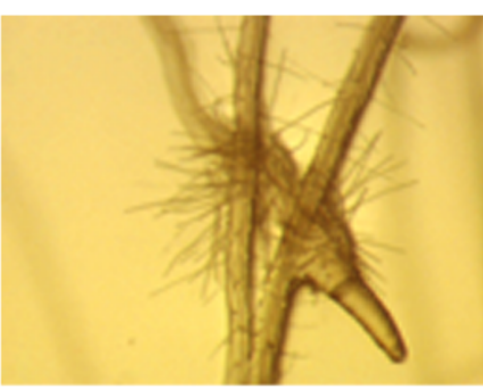

C

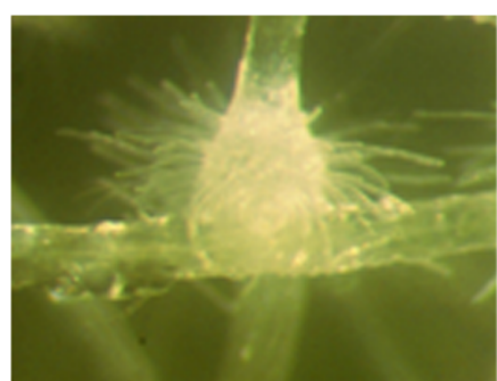

D

\section{Figure I}

Photos of Triphysaria haustoria with Arabidopsis host roots. A. Physical contact between parasite and host roots was made by laying Arabidopsis seedlings across the roots of $T$. versicolor seedlings in vitro. RNA for the host-induced library was isolated from the Triphysaria roots up to five hours after contact with Arabidopsis. B and C. Haustorium development on Triphysaria roots after 36 hours contact with Arabidopsis. $C$ shows a single Triphysaria root forming haustoria on two different Arabidopsis roots. D. Haustorium development after $24 \mathrm{hrs}$ exposure to $30 \mu \mathrm{M}$ DMBQ. 
Table I: EST and contig statistics of HF, HR and EDIT libraries

\begin{tabular}{cccccc}
\hline & Total ESTs & Assembled Contigs & Assembled Singlets & Total assemblies & $\begin{array}{c}\% \text { with AT } \\
\text { annotation' }\end{array}$ \\
\hline HF & 3386 & 673 & 401 & 1074 & $82 \%$ \\
HR & 3428 & 781 & 563 & 1344 & $80 \%$ \\
EDIT & 2216 & 403 & 999 & 1402 & $85 \%$ \\
\hline
\end{tabular}

I AT annotations were determined from BLASTX comparisons with ATHI.pep_cm_20040228 with a cutoff of e $\leq$ I0-8

are derived from those present in autotrophic angiosperms where they fulfill functions unrelated to parasitism. The identification of genes associated with haustorium development will provide insights into the evolutionary origins of plant parasitism. These genes will also elucidate the degree to which haustoria in different parasitic families are encoded by convergent or homologous genetic pathways.

Parasitic plants in the Orobanchaceae develop haustoria on their roots in response to contact with host roots. Several molecules, typically products of the phenylpropanoid pathway, have been identified that induce haustorium development when applied to Orobanchaceae roots in vitro [5,8-10]. Early haustorium development in response to exogenous signal molecules is characterized by three visible phenotypes: temporary cessation in root elongation, isodiametric cortical swelling, and haustorial hair proliferation $[11,12]$.

Molecular phylogeny places the Orobanchaceae on a single phylogenetic clade of parasites distinct from the nearest non-parasitic relative [13]. This suggests that the genetic mechanisms controlling haustorium development in the Orobanchaceae are likely similar. Triphysaria, formerly Orthocarpus, is an Orobanchaceae that grows as a common, springtime annual throughout the Pacific coast from Canada to Baja [14]. Triphysaria is a small genus of five intercrossing diploid species that are amenable to classical genetic analyses [15]. Triphysaria is closely related to the devastating agricultural weeds Striga and Orobanche; however, Triphysaria itself has no agricultural significance. Triphysaria are facultative parasites that can grow to maturity without host plants but will readily parasitize many host species when available, including Arabidopsis and maize. Triphysaria form haustoria within twelve hours of being exposed to Arabidopsis roots or root factors in vitro [16]. The speed, synchrony, and dependence on exogenous inducer makes haustorium development in Orobanchaceae an excellent system for identifying transcripts associated with subterranean plant-plant communications.

Towards the goal of identifying genes associated with plant parasitism, we sequenced cDNA libraries enriched by suppression subtractive hybridization (SSH) [17] for transcripts regulated in Triphysaria roots during haustorium development. To date we have sequenced approximately nine thousand ESTs from three SSH libraries generated after treating Triphysaria roots with either intact Arabidopsis roots or the chemical haustorium inducer 2,6dimethoxybenzoquinone (DMBQ). DMBQ, first purified as a haustorium inducer from sorghum [9], induces high rates of haustorium development in Triphysaria; however its role in mediating haustorium formation in Triphysaria - Arabidopsis interactions is not known. The Pscroph database provides on-line access to these EST and assembly sequences and provides BLAST and keyword search functions [18]. Comparative analysis with other transcriptomes will highlight genes and pathways associated with the origins of haustorium development and the evolution of heterotrophy in plants. These studies may provide insights into genetic strategies for developing crops resistant to parasitic weeds and into strategies for exploiting allelopathic interactions in agriculture generally.

\section{Construction and content Parasite treatments}

Triphysaria versicolor seeds were collected from thousands of cross-pollinating plants growing in a grassland stand near Napa CA. They were surface sterilized in a solution of $2 \%$ sodium hypochlorite (50\% household bleach) and $0.01 \%$ Triton $\mathrm{X}-1000$, rinsed thoroughly with water and germinated at $16^{\circ} \mathrm{C}$ in $0.25 \mathrm{X}$ Hoagland's solution and $1 \%$ agar [19]. After two to three weeks the seedlings were transferred along one edge of a square Petri dish containing the appropriate media and incubated at $25^{\circ} \mathrm{C}$ at a near vertical angle so that the roots grew down along the surface of the agar. Arabidopsis Columbia seeds were obtained from Lehle seeds (Round Rock, TX, USA), surface sterilized, and germinated in agar. Arabidopsis seedlings were then cultivated hydroponically for 40 days in liquid media ( 30 seedlings in $30 \mathrm{ml}$ of $0.25 \mathrm{X}$ Hoagland's media in $250 \mathrm{ml}$ flasks shaking at $50 \mathrm{rpm}$ at $25^{\circ} \mathrm{C}$ under a 16 hours light/8 hours dark cycle).

Haustorium development was induced five to seven days after the transfer of Triphysaria seedlings to vertical square plates by exposing their roots to Arabidopsis. This was done by placing roots of forty day old hydroponically grown 
Table 2: Localization of SSH products to gene regions '

\begin{tabular}{|c|c|c|c|c|c|}
\hline Library & Only coding & Only 5 ' non-coding & Only 3 ' non-coding & $\begin{array}{c}\text { Both } 5^{\prime} \text { and } 3^{\prime} \text { non- } \\
\text { coding }\end{array}$ & $\begin{array}{c}\text { Total SSH products } \\
\text { analyzed } 2\end{array}$ \\
\hline $\mathrm{HF}$ & 1302 (66\%) & $80(4 \%)$ & $566(29 \%)$ & 19 (1\%) & 1967 \\
\hline $\mathrm{HR}$ & $1212(54 \%)$ & $101(4 \%)$ & $868(39 \%)$ & $5 I(2 \%)$ & 2232 \\
\hline EDIT & 721 (38\%) & $428(22 \%)$ & $579(30 \%)$ & |7| (9\%) & 1899 \\
\hline
\end{tabular}

ISSH sequences were mapped to the plant gene identified as the best hit in BLASTX searches as described in the text. The number of produces predicted to contain non-coding sequences (3', 5', or both), or only coding sequences, are shown for each library.

2 Redundant ESTs within each library were identified from the BLASTX reports and removed from the analysis. ESTs lacking BLASTX hits with E values $\leq 10^{-8}$ were also eliminated from this analysis.

Arabidopsis across the roots of Triphysaria seedlings as shown in figure $1 \mathrm{a}$. Two $\mathrm{ml}$ of $0.25 \mathrm{X}$ Hoagland's media were applied to the roots to ensure good contact. Contact was maintained for up to five hours during which time Triphysaria roots were harvested for RNA. Haustoria were also induced by applying two $\mathrm{ml}$ of $10 \mu \mathrm{M}$ DMBQ directly to Triphysaria roots for construction of the EDIT library.

Early haustorium development could be detected within $24 \mathrm{hr}$ of host parasite contact as swollen, hairy knobs emerging just proximal to the Triphysaria root tips (Figure 1b,c,d). Triphysaria exposed to media without Arabidopsis or DMBQ did not develop haustoria.

\section{Library construction}

Triphysaria roots were dissected and frozen in liquid nitrogen at various time intervals ranging from immediately after treatment to up to five hours later. Triphysaria RNA was prepared as described [20]. Suppressive subtractive hybridization was used to generate cDNA libraries enriched for up or down regulated transcripts using a commercial kit (BD Sciences, Clontech, Mountain View, CA). The Host Forward (HF) library was enriched for transcripts upregulated in Triphysaria roots after contact with Arabidopsis and was made using mock-treated Triphysaria as the hybridization driver. The Host Reverse (HR) library was enriched for transcripts down regulated after host contact and was made using Arabidopsis exposed Triphysaria as driver. The EDIT library was enriched for transcripts upregulated in root tips within five hours of exposure to DMBQ as previously described [20].

\section{EST sequencing and assembly}

Subtracted cDNAs were ligated into pCR2.1-Topo (Invitrogen, Carlsbad, CA) and cloned in E. coli. Colonies were picked into 384 well microtiter plates and frozen at $80^{\circ} \mathrm{C}$. Sequencing templates were prepared from the library using a rolling circle amplification technology with TempliPhi kit (Amersham Biosciences, Piscataway, NJ). Sequencing reactions were run using the BigDye terminator v3.1 (Applied Biosystems, Foster City, CA.) and the products were separated and detected using the ABI3730xl DNA Fragment Analyzer (Applied Biosystems, Foster City,
Foster City, CA) at the UC Davis College of Agriculture and Environmental Sciences Genomics Facility.

DNA trace files were base-called using Phred (version $0.990722 . g$ ) and low quality sequences were removed based on a Phred p value $\leq 0.05$ [21]. The sequences were masked for the pCR 2.1-TOPO cloning vector, linker sequences, and repetitive sequences (excluding poly A and poly $\mathrm{T}$ ) based on alignments generated by the BLASTN program as used by the PyMood Sequence Processor (Allometra, Davis, CA) (Alexander Kozik, pers. comm.). Sequences less than 100 nts were discarded from further analyses. Approximately five percent of the clones had linker sequences internal to an ORF sequence. These were determined to be chimeras generated by the ligation of multiple SSH fragments into a single plasmid. The chimeric sequences were computationally digested into independent ESTs. The finished ESTs were submitted to GenBank's dbEST repository [22].

FASTA files of the finished ESTs were assembled into contigs using the cap3 program. Because we assembled trimmed and masked FASTA sequences, quality files were not included and the cap3 clipping function was unnecessary. The assembly was performed at the default parameters (overlap length cutoff $=30$; overlap percent identify cutoff $=75$; and overlap similarity score cutoff $=500$ ) [23]. Fifty percent of the assemblies were comprised of a single EST; an additional forty percent were comprised of two, three, or four ESTs. The assembly process identified about 1100 transcript assemblies in the HF library, 1300 in the HR library and 1400 in the EDIT library (Table 1).

\section{Database of early haustorial transcripts}

The EST sequences and assembly alignments are available at the Pscroph database [18]. Data are stored in a MySQL database and made available on the web using a phpMyAdmin interface. The database is housed at the University of California-Davis Genome Center.

Proteins predicted to be encoded by the assemblies were annotated from the BLASTX reports comparing Triphysaria sequences to either all proteins in GenBank (rel145.fsa_aa 

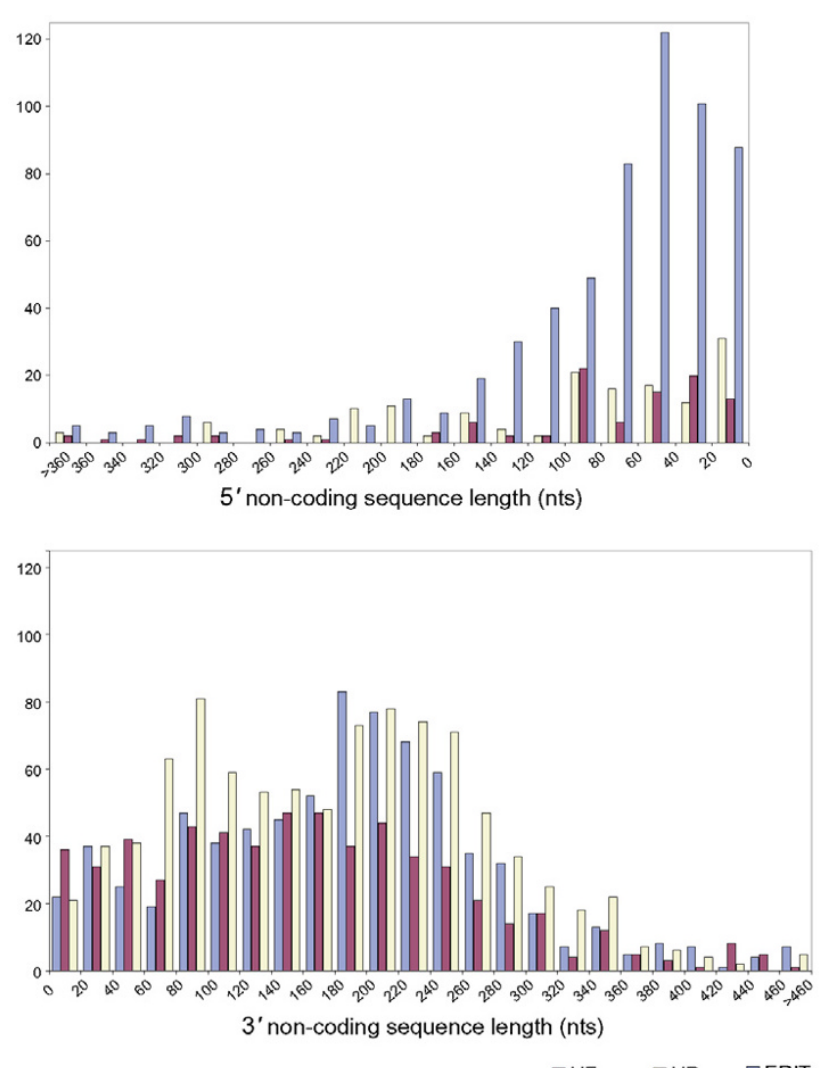

Figure 2

Length of 5 ' and 3 ' non-coding sequences. Protein translations of the SSH sequences in each library were mapped by BLASTX to the most homologous plant protein in GenBank. The histogram depicts the number and size of SSH sequences predicted to extend into either the $5^{\prime}$ or $3^{\prime}$ non-coding regions.

release Dec 15, 2004) or to all predicted proteins in Arabidopsis (ATH1.pep_cm_20040228). These BLAST reports can be accessed at the web site as full text files or by keyword searches of protein annotations. The keyword search function reports the best three hits obtained from GenBank or TAIR databases with e values $\leq 10^{-8}$. Each best hit is hyperlinked to the corresponding report page at NCBI or TAIR. The web site also provides a BLAST function that allows homology searches against DNA or protein sequences in each or all libraries.

\section{Utility and discussion SSH libraries}

We previously published a sequence characterization of 246 cDNAs from the EDIT library [20]. At that time we sequenced clones that had been selected by colony hybridizations for transcripts most differentially abun-

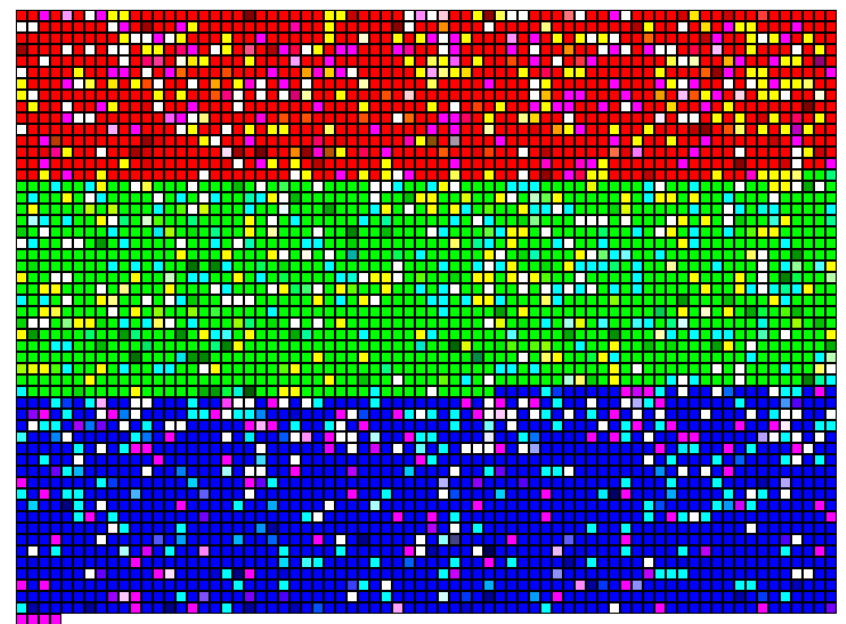

Figure 3

Virtual cDNA arrays and clone redundancy in different libraries. A FASTA file containing all HF, HR and EDIT assembly sequences was used as the target in BLASTN comparisons with sequences from each library as query. Each target cDNA (3820 total) was assigned a color based on homology to sequences in different libraries; sequences hybridizing to HF probes were assigned red, those hybridizing to HR probes green, and those with EDIT probes blue. The color intensity reflected the BLASTN score with higher values assigned to greater homology. Colors were mixed when sequences were present in more than one library: those present in both the HF and HR libraries were yellow, in both the HF and EDIT libraries pink, and in the HR and EDIT libraries teal. Assembly sequences with homologies in all three libraries are represented as white.

dant in the forward hybridization reaction compared to the reverse. This is suggested by the manufacturer to reduce the number of false positives. The colonies not analyzed fell into two, roughly equal sized groups; those that hybridized to both forward and reverse probes and those that hybridized to neither. While this step reduces the number of false positives, it also may eliminate interesting transcripts. In particular, colonies that hybridized with neither probe likely represented weakly expressed, low abundance transcripts [24]. Furthermore, colonies that hybridized to probes from multiple libraries may contain conserved domains in otherwise distinct proteins. Therefore we sequenced additional, unselected clones from the EDIT library and eliminated the hybridization in constructing the HF and HR libraries.

The SSH procedure included an Rsa 1 digestion step prior to cloning that resulted in bidirectional cloning and in single transcripts being represented by multiple, nonoverlapping SSH products. In order to determine the distribution of SSH products relative to the $3^{\prime}$ and $5^{\prime}$ ends of 
Table 3: Occurrence of transcripts in different libraries 1

\begin{tabular}{cccc}
\hline Category & Total \# assemblies & \# assemblies in category & \% category specific \\
\hline HF specific & 1,074 & 702 & $65 \%$ \\
HR specific & 1,344 & 910 & $68 \%$ \\
EDIT specific & 1,402 & 1,018 & $73 \%$ \\
HF+HR & 2,418 & 213 & $9 \%$ \\
HF+EDIT & 2,476 & 180 & $7 \%$ \\
HR+EDIT & 2,746 & 218 & $8 \%$ \\
HF+HR+EDIT & 3,820 & 92 & $2 \%$ \\
\hline
\end{tabular}

IBLASTN $\left(\mathrm{e} \leq 10^{-20}\right)$ was used to identify assemblies found in one or more libraries.

the encoding gene, we mapped the virtual translations of the SSH ESTs onto the most homologous protein in the plant protein database. The tcl_blast_parser_123_V017 was used to convert BLASTX output data to a table format suitable for manipulation in a spreadsheet [25]. Using the length of the target ORF and the amino acid locations corresponding to the start and stop of the aligned region between the SSH and plant homologs, we estimated the number and length of Triphysaria sequences predicted to be either $3^{\prime}$ or $5^{\prime}$ non-coding (Table 2, Figure 2). These regions provide good candidate sites for identifying gene specific primers.

Depending on the library, from $34 \%$ to $62 \%$ of the Triphysaria sequences were predicted to include non-coding sequences; one to ten percent of the cDNAs included both $5^{\prime}$ and $3^{\prime}$ non-coding sequences (Table 2 ). There were more $3^{\prime}$ than $5^{\prime}$ non-coding sequences in all libraries; there were eight times more $3^{\prime}$ sequences in the HR library. The 3' non coding regions recovered in the SSH libraries were also longer than those predicted for the $5^{\prime}$ (Figure 2). The 3' bias likely results from the initial cDNA synthesis reaction that is primed with poly-T. Depending on the library, between ten and twenty percent of the SSH products had poly-A tracts. The predominance of ORF encoded sequences in the libraries demonstrates that these libraries were less biased towards 3' sequences than would be expected without the Rsa 1 digestion.

\section{Interlibrary comparisons}

We used BLASTN to identify nucleotide sequences in common between the different libraries. This is a bioinformatics alternative to colony hybridization to identify interlibrary sequence homologies. Figure 3 is a color representation of the BLASTN results generated by the PyMood software package (Allometra, Davis, CA). The squares represent 3820 assembly sequences arrayed in the order HF, HR and EDIT. BLASTN was performed using the concatenated sequences from the virtual array as target and sequences from each library as query. PyMood parsed the BLAST output and assigned mixes of red, blue and yel- low colors to each sequence based on the degree to which the target sequence had homologies in other libraries. The intensity of color was a function of the BLASTN e value and colors were mixed when sequences were present in more than one library.

As shown in table 3, about seventy percent of the sequences were specific to a single library. About seven percent of the assemblies were found in both HF and EDIT libraries but not HR; these represent likely candidates for early haustorium development. However similar numbers of sequences were in common between the HF and HR libraries, indicating a basal level of interlibrary redundancy. The number of sequences in common between forward and subtracted libraries is higher than expected if there was no selection for particular crosshybridizing sequences. If the one thousand sequenced assemblies in each library represent $2 \%$ of the approximately 20,000 root transcripts [26], about $0.4 \%$ of assemblies would be expected in both libraries by chance alone. In a previously published wet lab characterization of the EDIT library, we reported that about $20 \%$ of the clones cross-hybridized to transcripts in both forward and reverse subtracted probes. Other experiments employing SSH procedures report false positive rates of cross-hybridizing clones of $30-50 \%[27,28]$. The Clonetech Selelect PCR users guide states that recovery rates of false positives will vary between tissue types and RNA preparations [29].

The unpredictably high rate of cross-library hybridizing transcripts was not a function of the assembly because a BLASTN analysis of EST sequences before assembly gave similar results (data not shown). Approximately half of the cross-hybridizing sequences had multiple sequence polymorphisms, suggesting these are alleles of coexpressed genes or domains. The levels of expression of cross-hybridizing sequences were estimated from the Arabidopsis MPSS database to determine if they are particularly highly expressed in roots [26]. The Arabidopsis homologs to cross-hybridizing Triphysaria sequences ranged in their root expression between six and over three 
Table 4: Functional classification of library specific sequences '

\begin{tabular}{lrrr}
\hline Biological function & HF vs HR & HF vs EDIT & HR vs EDIT \\
\hline cell organization and biogenesis & 0.87 & 0.13 & 0.43 \\
developmental processes & 0.82 & 0.18 & 0.31 \\
DNA or RNA metabolism & $4.26 *$ & 2.25 & $4.96 *$ \\
electron transport or energy pathways & $11.24 * * *$ & $4.35 *$ & 0.99 \\
protein metabolism & $8.42 * *$ & 1.31 & 0.17 \\
response to abiotic or biotic stimulus & 2.20 & 2.19 & 0.73 \\
response to stress & $4.87 *$ & 0.03 & 0.60 \\
signal transduction & 0.27 & 0.02 & 0.12 \\
transcription & 0.21 & 0.12 & \\
transport & 3.15 & 0.84 & 2.51 \\
other biological processes & 0.37 & 0.05 & 2.80 \\
other cellular processes & 1.99 & 0.05 & 3.31 \\
other metabolic processes & 1.05 & 1.93 \\
other physiological processes & 1.73 & 0.24 & $3.99 *$ \\
biological process unknown & 0.07 & 1.58 & 2.72 \\
\hline
\end{tabular}

IThe proportion of clones in each library assigned a particular GO function were compared between different libraries and chi square used to indicate significance differences in functional representation between libraries (*** $p \leq 0.001$, ** $p \leq 0.01$, * $p \leq 0.05$ ).

thousand transcripts per trillion. An ANOVA analysis indicated no significant differences between the predicted expression levels of library specific sequences from false positives present in both forward and subtracted libraries (data not shown).

One possible explanation for the unpredictably large number of false positive clones following SSH procedures is miss-priming at the first or second PCR reactions. cDNAs that were not selected during hybridization would be similarly amplified from both libraries if they have sufficient homology to the 22-mers used in the final amplifications prior to cloning.

\section{Functional classifications}

BLASTX was used to assign putative functions to virtual translations of each library specific assembly. Roughly $75-80 \%$ of the library specific sequences had homologies in the Arabidopsis protein database at an e value $\leq 10^{-8}$. Using the AT number of the best Arabidopsis hits, the putative Triphysaria proteins were placed into functional categories using the Gene Ontology at TAIR [30]. The GO terms obtained for each library are summarized in supplemental table 1. The TAIR output included multiple GO terms for most assemblies so there are more GO descriptors than transcripts.

We used chi squared analyses to determine whether different libraries were enriched for certain GO functional categories (supplemental table 1). The frequency of a particular GO term was determined from the total number of GO terms obtained for that library. The relative frequencies of specific GO terms were then compared between libraries. Table 4 summarizes pair wise comparisons between libraries in the proportion of transcripts in each of the GO categories. The most significant functional enrichment was the overrepresentation of transcripts associated with electron transport in the HF library relative to HR (table 4). Electron transport functions were also enriched in the EDIT library relative to HR although at a lower significance ( $\mathrm{p} \leq 0.05)$. Correspondingly, transcripts associated with mitochondria were also over-represented in the HF and EDIT libraries relative to the HR library. The over representation of transcripts associated with electron transport is consistent with the model that haustorial inducing factors trigger development through redox mediated mechanisms [31,32]. The HF library was also enriched for transcripts associated with stress responses. This was previously recognized in the EDIT library and is consistent with the long standing hypothesis that parasitic plants recruit defense related genes for host recognition $[7,20]$.

Transcripts associated with the metabolism of nucleic acids and proteins were significantly less abundant in the HF and EDIT compared to the HR libraries. The down regulation of DNA metabolism genes is consistent with the earlier observations that cell division and DNA synthesis is rapidly terminated in Striga upon contact with DMBQ [33]. There were also fewer transcripts predicted to encode protein metabolism functions in the HF and EDIT libraries. While changes in protein profiles have been observed in Striga following DMBQ treatment, the overall reduction in the proportion of transcripts encoding protein metabolism genes was not expected [34,35].

\section{Conclusion}

Parasitic plants provide an excellent system for studying genetic mechanisms of chemical signaling between plants. In addition, parasitic weeds are among the world's 
most destructive agricultural pests against which few genetic resistances are available. Genetic suppression of parasite development at early stages in parasitism is a promising approach for engineering resistance against parasitic weeds but requires knowledge of the genetic factors regulating parasite development. The Pscroph database contains parasitic plant transcripts regulated by host encoded factors; these provide potential points for engineering parasite resistance. More generally, the identification of regulatory elements induced by the presence of other plants provides the potential for genetic weed control strategies.

\section{Availability and requirements}

The Pscroph database can be accessed at http:// Pscroph.ucdavis.edu.

\section{Authors' contributions}

MJT developed the EST and assembly pipeline and the Pscroph database, AAT and NT made the SSH libraries and prepared them for sequencing, RLR mapped the SSH products to ORFs and manages the Pscroph databases, JIY designed and coordinated the project and wrote the manuscript with input and editing from the other authors. All authors read and approved the final manuscript.

\section{Additional material}

\section{Supplementary files}

All additional files are in Excel format. Footnote to additional files 1-7: Sequences from three libraries were compared by BLASTN to determine which sequences were present in more than one library. The BLASTN cutoff e value was $10^{-80}$. Supplemental tables 1-3 contain the library specific sequences and their annotations, tables 4- 6 contain sequences present in HF and HR libraries, or HF and EDIT libraries, or HR and EDIT libraries, and table 7 contains sequences present in all three libraries. The annotations shown are parsed from a BLASTX search of the Arabidopsis protein database ATH1.pep_cm_20040228. Footnote to additional file 8: The HF, HR and EDIT specific sequences were annotated with Gene Ontogeny descriptors by cross referencing to the best BLASTX hit in ATH1.pep via TAIR [30]. The first three columns of numbers show the number of times a particular GO descriptor was assigned. Most of the assemblies were annotated with multiple GO descriptors so there are more descriptors than assemblies. A total of $5499 \mathrm{GO}$ descriptors were obtained for the HF library, 7222 GO descriptors from the HR and 8381 descriptors from the EDIT library. Pair-wise comparisons were made between each library for the relative proportion of annotations in a particular GO class. For example, $5499 \mathrm{GO}$ annotations were obtained from the HF assemblies; of these, $666(12.1 \%)$ were annotated as "other physiological processes". Similarly, 931 of the 7222 (12.9\%) of the GO annotations obtained from the HR library were "other physiological processes". The resultant Chi-squared value is 1.73 , suggesting there are not significant differences in the proportion of assemblies annotated as "other physiological processes" between the HF and HR libraries. In contrast, the relative proportion of "other physiological processes" annotations in the EDIT library is $11.8 \%$ (992 out of 7222 total); this is significantly different from the proportions obtained for the HR library (12.9\%) but not the HF $(12.1 \%)$ at $p \leq 0.05$. Numbers in the cells are the Chi-squared values calculated. The yellow shaded cells indicate significant $p$ values $\leq 0.05$.
Additional file 1 - HF specific assemblies

Additional file 2 - HR specific assemblies

Additional file 3 - EDIT specific assemblies

Additional file 4 - Assemblies in both HF and HR

Additional file 5 - Assemblies in both HF and EDIT

Additional file 6 - Assemblies in both HR and EDIT

Additional file 7 - Assemblies in HF, HR and EDIT

Additional file 8 - GO category analysis

[http://www.biomedcentral.com/content/supplementary/1471-

2229-5-24-S1.xls]

\section{Acknowledgements}

The authors recognize and sincerely appreciate the contributions of Marta Matvienko, Alexander Kozik, and Steve Edberg to the development of this database. The database is housed at the University of California-Davis Genome Center. This work was supported by NSF grant \# 0236545. M. J. Torres was a recipient of an NIH Biotechnology Training Grant Award.

\section{References}

I. Kuijt J: The Biology of Parasitic Flowering Plants. Berkeley, University of California Press; 1969:246.

2. Press MC, Graves JD: Parasitic Plants. London, Chapman and Hall; 1995:292.

3. Parker C, Riches CR: Parasitic Weeds of the World: Biology and Control. Wallingford, CAB International; 1993:332.

4. Nickrent D: Parasitic Plant Connection. [http://www.parasitic plants.siu.edu].

5. Riopel JL, Timko MP: Haustorial initiation and differentiation. In Parasitic Plants Edited by: Press MC and Graves JD. London, Chapman and Hall; 1995:39-79.

6. Nickrent DL, Duff RJ, Colwell AE, Wolfe AD, Young ND, Steiner KE, dePamphilis CW: Molecular phylogenetic and evolutionary studies of parasitic plants. In Molecular Systematics of Plants II DNA Sequencing Edited by: Soltis DE, Soltis PS and Doyle JJ. Boston, Kluwer Academic Publishers; 1998:211-24I.

7. Atsatt PR: Parasitic flowering plants: How did they evolve? Amer Nat 1973, 107:502-510.

8. Steffens JC, Lynn DG, Kamat VS, Riopel JL: Molecular specificity of haustorial induction in Agalinis purpurea (L.) Raf. (Scrophulariaceae). Annals of Botany 1982, 50: I-7.

9. Chang M, Lynn DG: The haustorium and the chemistry of host recognition in parasitic angiosperms. Journal of Chemical Ecology 1986, I 2:561-579.

10. Albrecht $\mathrm{H}$, Yoder JI, Phillips DA: Flavonoids promote haustoria formation in the root parasite Triphysaria. Plant Physiology 1999, I 1 9:585-59|.

II. Okonkwo SNC, Nwoke FIO: Initiation, development and stucture of the primary haustorium in Striga gesnerioides (Scrophulariaceae). Annals of Botany 1978, 42:455-463.

12. Baird WV, Riopel JL: Experimental studies of haustorium initiation and early development in Agalinis purpurea (L.) Raf. (Scrophulariaceae). American Journal of Botany 1984, 71:803-814.

13. dePamphilis CW, Young NN, Wolfe AD: Evolution of plastid gene rps2 in a lineage of hemiparasitic and holoparasitic plants: Many losses of photosynthesis and complex patterns of rate variation. Proc Natl Acad Sci USA 1997, 93:7367-7372.

14. Hickman JC: The Jepson Manual; Higher Plants of California. Berkeley, CA, University of California Press; 1993:1400.

15. Yoder Jl: Self and cross-compatibility in three species of the hemiparasite Triphysaria (Scrophulariaceae). Environ Exp Bot 1998, 39:77-83.

16. Goldwasser Y, Westwood JH, Yoder Jl: The use of Arabidopsis to study interactions between parasitic angiosperms and their plant hosts. In The Arabidopsis Book Edited by: Somerville $C$ and Meyerowitz E. Rockville, MD, American Society Plant Biologists; 2002.

17. Diatchenko L, Lau YF, Campbell AP, Chenchik A, Moqadam F, Huang B, Lukyanov S, Lukyanov K, Gurskaya N, Sverdlov ED, Siebert PD: Suppression subtractive hybridization: a method for gener- 
ating differentially regulated or tissue-specific cDNA probes and libraries. Proc Natl Acad Sci U S A 1996, 93:6025-6030.

18. Pscroph: A parasitic plant database. [http://
[ pscroph.ucdavis.edu].

19. Jamison DS, Yoder Jl: Heritable variation in quinone-induced haustorium development in the parasitic plant Triphysaria. Plant Physiol 200I, 125: I870-1879.

20. Matvienko M, Torres MJ, Yoder Jl: Transcriptional responses in the hemiparasitic plant Triphysaria versicolor to host plant signals. Plant Physiol 200I, I 27:272-282.

21. Ewing B, Hillier LD, Wendl MC, Green P: Base-calling of automated sequencer traces using phred. I. Accuracy assessment. Genome Research 1998, 8:175-185.

22. Boguski MS, Lowe TM, Tolstoshev CM: dbEST--database for expressed sequence tags. Nature Genetics 1993, 4:332-333.

23. Huang $X Q$, Madan A: CAP3: A DNA sequence assembly program. Genome Res 1999, 9:868-877.

24. Florian F, Adam K, Martin. C: Identification of novel putative regulatory genes induced during alfalfa nodule development with a cold-plaque screening procedure. Molecular Plant-Microbe Interactions 1998, I I:358-366.

25. Kozik A, Chan B, Michelmore R: How to parse results of BLAST search (ESTs vs proteins) and set up tables for mySQL database. [http://cgpdb.ucdavis.edu/BlastParser/Blast Parser 017.html].

26. Meyers BC, Lee DK, Vu TH, Tej SS, Edberg SB, Matvienko M, Tindell LD: Arabidopsis MPSS. An online resource for quantitative expression analysis. Plant Physiol 2004, I35:80I-8I3.

27. Ji SJ, Lu YC, Feng JX, Wei G, Li J, Shi YH, Fu Q, Liu D, Luo JC, Zhu $Y X$ : Isolation and analyses of genes preferentially expressed during early cotton fiber development by subtractive PCR and cDNA array. Nucl Acids Res 2003, 3 I:2534-2543.

28. Zheng J, Zhao J, Tao Y, Wang J, Liu Y, Fu J, Jin Y, Gao P, Zhang J, Bai $Y$, Wang G: Isolation and analysis of water stress induced genes in maize seedlings by subtractive PCR and cDNA macroarray. Plant Molecular Biology 2004, 55:807-823.

29. Clontech: PCR-Select Differential Screening Kit, Users manual PT 3I38-I. [http://www.clontech.com/techinfo/manuals/PDF/ PT3138-I.pdf].

30. TAIR: Gene Ontology at TAIR: The Arabidopsis Information Resource. [http://www.arabidopsis.org/tools/bulk/go/index.jsp].

3I. Smith CE, Ruttledge T, Zeng Z, O'Malley RC, Lynn DG: A mechanism for inducing plant development- the genesis of a specific inhibitor. Proceedings National Academy of Sciences USA 1996, 93:6986-6991.

32. Matvienko M, Wojtowicz A, Wrobel R, Jamison D, Goldwasser $Y$, Yoder Jl: Quinone oxidoreductase message levels are differentially regulated in parasitic and non-parasitic plants exposed to allelopathic quinones. Plant Journal 200I, 25:375-387.

33. Keyes WJ, O'Malley RC, Kim D, Lynn DG: Signaling organogenesis in parasitic angiosperms: Xenognosin generation, perception, and response. J Plant Growth Regul 2000, V 19:2 17-23I.

34. Wolf SJ, Timko MP: Analysis of in vivo protein sythesis and histological studies of haustorial formation in root cultures of witchweed (Striga asiatica L. Kuntze). J Exp Bot 1992, 43:1339-1348.

35. Stranger A, Murphy A, Corbett JM, Dunn MJ, Bolwell GP, Stewart GR: Changes in patterns of protein synthesis during haustorial development of Striga hermonthica (Del.) Benth. seedlings. Journal of Experimental Botany 1995, 46:277-283.
Publish with Bio Med Central and every scientist can read your work free of charge

"BioMed Central will be the most significant development for disseminating the results of biomedical research in our lifetime. "

Sir Paul Nurse, Cancer Research UK

Your research papers will be:

- available free of charge to the entire biomedical community

- peer reviewed and published immediately upon acceptance

- cited in PubMed and archived on PubMed Central

- yours - you keep the copyright
BioMedcentral 